\title{
RESPONSE SURFACE METHODOLOGY ANALYSIS OF POLYPHENOL RECOVERY FROM ARTICHOKE WASTE
}

\author{
Antonio Zuorro \\ Department of Chemical Engineering, Materials and Environment, Sapienza University, Rome, Italy
}

Received 2014-05-14; Revised 2014-06-10; Accepted 2014-07-03

\begin{abstract}
Large amounts of a solid waste consisting mainly of outer bracts and stems are produced from the industrial processing of artichokes. In this study, the recovery of polyphenols from the two waste components was investigated. Extraction experiments were carried outby an environmentally friendly procedure using aqueous ethanol as solvent. The total polyphenol content, expressed as mg of GAE per g of dry weight, was $10.23 \pm 0.68$ $\mathrm{mg} / \mathrm{g}$ for bracts and $16.36 \pm 0.85 \mathrm{mg} / \mathrm{g}$ for stems. To evaluate the effect of Temperature (T), Extraction time (E) and liquid-to-solid Ratio (R) on the extraction yields, a central composite design coupled with response surface methodology was used. Under the best conditions $\left(\mathrm{T}=50^{\circ} \mathrm{C}, \mathrm{E}=110.4 \mathrm{~min}\right.$ and $\left.\mathrm{R}=20 \mathrm{~mL} \mathrm{~g}^{-1}\right)$, extraction yields between 90 and $93 \%$ were obtained. Statistical analysis of the data showed that $\mathrm{E}$ was the most influential factor, followed by T and R. Simplified polynomial models were developed to describe the effect of individual factors and their interactions on the extraction yield of polyphenols. Overall, the results of this study support the potential of using artichoke waste as a source of natural phenolic antioxidants and give useful directions on how to improve recovery by proper selection of extraction conditions.
\end{abstract}

Keywords: Artichoke Waste, Polyphenols, Extraction, Optimization, Response Surface Methodology

\section{INTRODUCTION}

Globe artichoke (Cynarascolymus L.), a perennial plant originating from the Mediterranean region, is commercially grown for its edible buds. Italy is the world's largest producer of artichokes, with an annual production of over 400,000 tons (FAOSTAT, 2011).

From the industrial processing of artichokes, large amounts of solid and liquid wastes are generated. Solid residues constitute about $80-85 \%$ of the total plant biomass and consist mainly of the stems and the external parts of the flowers, known as bracts. This material is not suitable for human consumption and is usually discarded. Waste disposal represents an additional cost to the producers and contributes to the environmental impact of this industrial activity.

Over the last years, attempts have been made to find possible uses for artichoke waste. Raccuia et al. (2013) examined the possibility of using artichoke residues for energy or paper-pulp production. In another study, the anaerobic digestibility of artichoke waste was investigated (Fabbri et al., 2014). High methane yields and efficient degradation of the biomass were observed, thus supporting its use for biogas production. López-Molina et al. (2005) proposed a method for extracting high-molecular-weight inulin from artichoke bracts. Inulin is a heterogeneous blend of fructose polymers widely found in nature as plant storage carbohydrates. Microbiological tests on cultures of Bifidobacterium spp. showed that artichoke inulin had beneficial prebiotic properties and could be used in food and pharmaceutical applications (López-Molina et al., 2005). Other studies evaluated the feasibility of usingthe enzyme peroxidase extracted from artichoke waste to remove phenols from wastewater (Sergio et al., 2010).

Recently, increasing interest has emerged in the search of natural antioxidants from vegetable and fruit wastes (Kosseva, 2009). The recovery of lycopene from tomato pomace (Zuorro et al., 2013b) or of phenolic compounds from spent coffee grounds (Panusa et al., 2013; Zuorro and Lavecchia, 2013) or bilberry processing waste (Zuorro and Lavecchia, 2014) are some examples of the applications under investigation. Due to its high antioxidant potential, resulting from the 
presence of high levels of polyphenols, artichoke waste is a promising candidate for this purpose. In addition, its wide availability and low cost of pretreatment make it suitable for industrial exploitation (Wang, 2013).

Polyphenols are a class of plant secondary metabolites with diverse biological functions, one of the most important of which is the protection against oxidative stress. The major polyphenols in artichoke tissues are caffeoylquinic acids, a group of esters of quinic and caffeic acids, but many other bioactives, such as luteolin and apigenin derivatives, are also present (Pandino et al., 2011). From the examination of the phenolic composition of six artichoke varieties in the Mediterranean region, Negro et al. (2012) found that chlorogenic acid, cynarin (1,5-dicaffeoylquinic acid), luteolin 7-O-rutinoside and luteolin 7-O-glucoside were the predominant polyphenols. Studies have also shown that theamount ofpolyphenols in artichokesmay vary significantly with developmental stage and genotype and that they tend to accumulate preferentially in different plant parts (Pandino et al., 2011; 2013).

Despite the presence of valuable health-promoting compounds in artichoke waste, little attention has so far been devoted to the technological aspects of their recovery. In particular, studies on the effects of process conditions on the extraction yields or on the optimization of the recovery process are currently lacking.

This study was aimed at investigating the influence of the main process variables on the recovery of phenolic compounds from artichoke waste. Polyphenols were extracted from the plant material by an environmentally friendly solvent-extraction procedure using aqueous ethanol as the solvent. The two main components of the waste, the outer bracts and the stems, were individually tested in order to highlight possible differences in their phenolic content and to assess whether the recovery of polyphenols could be affected by their different structural and physicochemical features. A rigorous approach based on factorial design and Response Surface Methodology (RSM) was used to analyze the process.

\section{MATERIALS AND METHODS}

\subsection{Chemicals and Reagents}

Ethanol (CAS 64-17-5), hydrochloric acid (CAS 7647-01-0) and sodium carbonate (CAS 497-19-8) were obtained from Carlo Erba (Milano, Italy). Gallic acid (3,4,5-trihydroxybenzoic acid, CAS 149-91-7) and the Folin-Ciocalteu reagent were purchased from SigmaAldrich Co. (St. Louis, Mo, USA). All chemicals were reagent grade and used without further purification. Demineralized water was used for artichoke blanching.

\subsection{Plant Material}

Fresh artichokes were purchased from a local market. Outer bracts and stems were removed by a knife, chopped and milled for a few minutesin an electric grinder (Moulinex, Italy). Then they were blanched in water at $85^{\circ} \mathrm{C}$ for $15 \mathrm{~min}$ in order to inactivate polyphenol oxidase, the enzyme responsible for the oxidation of polyphenols. Finally, the plant material was partially dried at $50^{\circ} \mathrm{C}$ in a forced-air dehydrator (Stöckli, Switzerland) and stored in the dark until use.

\subsection{Analytical Methods}

Moisture content was determined by an electronic moisture analyzer (model MAC 50/1, Radwag, Poland).

A three-stage extraction procedure was used to evaluate the initial phenolic content of artichokebracts and stems (Zuorro and Lavecchia, 2012). The assay was performed at $60^{\circ} \mathrm{C}$, using an extraction time of $30 \mathrm{~min}$ and a liquid-to-solid ratio of 100,50 and $25 \mathrm{~mL} \mathrm{~g}^{-1}$ in the first, second and third stage, respectively.

Total phenolics were determined by the FolinCiocalteu method with some modifications (Zuorro and Lavecchia, 2012). The results were expressed as gallic acid equivalents (mg GAE per g of dry solid) using a calibration curve obtained with gallic acid standards.

\subsection{Extraction Procedure}

Solvent extraction experiments were carried out in batch mode in magnetically stirred and thermostated $\left( \pm 0.1^{\circ} \mathrm{C}\right)$ screw-cap flasks, following the procedure described elsewhere (Zuorro and Lavecchia, 2011). Aqueous ethanol (50\% v/v) was used as the solvent. Briefly, $20 \mathrm{~mL}$ of solvent and the appropriate amount of plant material (roughly between 0.5 and $6.5 \mathrm{~g}$ ) were placed into the flask. At the desired time, a sample of the liquid was taken, passed through a $45 \mu \mathrm{m}$ nylon filter and assayed for total phenolics.

\subsection{Experimental Design}

A Central Composite Rotatable Design (CCRD) was used to evaluate the effects of Temperature (T), Extraction time (E), liquid-to-solid Ratio (R). The CCRD consisted of a full $2^{3}$ factorial design ( 8 runs), a replicated central point ( 6 runs) and axial points at distance $\pm \alpha$ from the central point ( 6 runs). To ensure rotatability and orthogonality of the design, the value of $\alpha$ was taken as $\left(2^{3}\right)^{1 / 4}=1.682$ (Montgomery, 2008). Factor levels were chosen to cover a range of values of practical interest and are reported, in natural and coded values, in Table 1. 
Table 1. Factors and levels of the experimental design ( $\mathrm{T}$ is the temperature, $\mathrm{E}$ the extraction time and $\mathrm{R}$ the liquid-to-solid ratio)

\begin{tabular}{|c|c|c|c|c|c|c|}
\hline \multirow[b]{2}{*}{ Factor } & \multirow[b]{2}{*}{ Unit } & \multicolumn{5}{|c|}{ Level } \\
\hline & & -2 & -1 & 0 & +1 & +2 \\
\hline $\mathrm{T}$ & ${ }^{\circ} \mathrm{C}$ & 33.2 & 40 & 50 & 60 & 66.8 \\
\hline $\mathrm{E}$ & $\min$ & 9.5 & 30 & 60 & 90 & 110.4 \\
\hline $\mathrm{R}$ & $\mathrm{mL} \mathrm{g}^{-1}$ & 3.2 & 10 & 20 & 30 & 36.8 \\
\hline
\end{tabular}

Coded values were obtained by the following transformations Equation 1 to 3:

$x_{1}=\frac{T-50}{10}$

$x_{2}=\frac{E-60}{30}$

$x_{3}=\frac{R-20}{10}$

The extraction yield of phenolic compounds (y), expressed as the percentage amount of extracted phenolics to the total amount of polyphenols in the waste, was used as the response variable. Overall, the experimental design consisted of 30 runs, which were conducted randomly to minimize the effects of uncontrolled factors (Table 2).

The design and analysis of experiments were performed using the statistical software Design-Expert ${ }^{\circledR}$, version 7.0.0 (Stat-Ease, Inc., Minneapolis, MN, USA).

\section{RESULTS}

\subsection{Characterization of Artichoke Waste}

The initial moisture content of artichoke waste was 83.4 $\pm 2.9 \mathrm{wt} \%$. After drying, the moisture content reduced to $6.23 \pm 0.73 \mathrm{wt} \%$. The polyphenol content, expressed as $\mathrm{mg}$ of GAE per $\mathrm{g}$ of dry weight, was $10.23 \pm 0.68 \mathrm{mg} / \mathrm{g}$ for bracts and $16.36 \pm 0.85 \mathrm{mg} / \mathrm{g}$ for stems. Thus, the amount of phenolic compounds in stems was about 1.5 times higher than in bracts, which is in line with the results reported in previous studies (Pandino et al., 2011; Negro et al., 2012; Gaafar and Salama, 2013).

\subsection{Model Fitting and Analysis of Response Surface}

Table 2 shows the results of the experimental design. It can be seen that the extraction yields were between
45.2 and $92.7 \%$ (average value: $66 \%$ ) for bracts and between 30.1 and $90.2 \%$ (average value: $55.6 \%$ ) for stems. For both materials, the maximum yield was achieved at $\mathrm{T}=50^{\circ} \mathrm{C}, \mathrm{E}=110.4 \mathrm{~min}, \mathrm{R}=20 \mathrm{~mL} \mathrm{~g}^{-1}$.

To evaluate the contribution of the three main factors T, E and Rto the extraction efficiency, the experimental data were correlated by different empirical polynomial models. These models are commonly used to describe the behavior of complex systems because of their good interpolation ability and ease of parameter estimation (Montgomery, 2008; Zuorro et al., 2013a). For bracts, the linear model as reported in Equation 4:

$y=\beta_{0}+\sum_{i=1}^{3} \beta_{i} x_{i}$

Provided the best fit to the data, while for stems the quadratic model described by Equation 5:

$y=\beta_{0}+\sum_{i=1}^{3} \beta_{i} x_{i}+\sum_{i=1}^{3} \beta_{i} x_{i}^{2}+\sum_{i=1}^{3} \sum_{j=1, i<j}^{3} \beta_{i j} x_{i} x_{j}$

Was more adequate. In the above Equation 4 and 5, $y$ is the process response, $x$ 's are the independent variables, $\beta_{0}$ is the intercept and $\beta_{\mathrm{i}}, \beta_{\mathrm{ii}}$ and $\beta_{\mathrm{ij}}$ are the linear, pure quadratic and interaction coefficients, respectively.

The unknown model coefficients were estimated by the least-squares method and are listed in Table 3 together with their standard errors and p-values. Both models provided a good fit to the data, with coefficient of determination $\left(\mathrm{R}^{2}\right)$ and adjusted- $\mathrm{R}^{2}$ equal to, respectively, 89.9 and $88 \%$ for bracts and 95.4 and $91.3 \%$ for stems. Analysis of residuals showed no apparent departures from basic ANOVA assumptions, i.e., normally distributed errors with constant variance and independent of one another. A comparison between experimental and calculated yields for the two plant materials is shown in Fig. 1. 
Table 2. Experimental design layout for polyphenol recovery from artichoke waste. SO denotes the standard order of runs

\begin{tabular}{|c|c|c|c|c|c|}
\hline \multirow[b]{2}{*}{ SO } & \multirow[b]{2}{*}{$x_{1}$} & \multirow[b]{2}{*}{$x_{2}$} & \multirow[b]{2}{*}{$x_{3}$} & \multicolumn{2}{|l|}{$y$} \\
\hline & & & & Bracts & Stems \\
\hline 1 & -1 & -1 & -1 & 45.16 & 31.91 \\
\hline 2 & 1 & -1 & -1 & 46.73 & 43.28 \\
\hline 3 & -1 & 1 & -1 & 73.61 & 63.02 \\
\hline 4 & 1 & 1 & -1 & 80.65 & 71.09 \\
\hline 5 & -1 & -1 & 1 & 56.99 & 47.07 \\
\hline 6 & 1 & -1 & 1 & 57.48 & 54.28 \\
\hline 7 & -1 & 1 & 1 & 81.62 & 61.74 \\
\hline 8 & 1 & 1 & 1 & 78.79 & 71.52 \\
\hline 9 & $-\alpha$ & 0 & 0 & 52.20 & 30.07 \\
\hline 10 & $+\alpha$ & 0 & 0 & 73.70 & 42.11 \\
\hline 11 & 0 & $-\alpha$ & 0 & 46.14 & 41.26 \\
\hline 12 & 0 & $+\alpha$ & 0 & 92.67 & 90.22 \\
\hline 13 & 0 & 0 & $-\alpha$ & 62.76 & 60.88 \\
\hline 14 & 0 & 0 & $+\alpha$ & 70.48 & 60.15 \\
\hline 15 & 0 & 0 & 0 & 72.83 & 60.51 \\
\hline 16 & 0 & 0 & 0 & 61.00 & 55.68 \\
\hline 17 & 0 & 0 & 0 & 69.70 & 57.95 \\
\hline 18 & 0 & 0 & 0 & 62.95 & 59.90 \\
\hline 19 & 0 & 0 & 0 & 67.94 & 49.76 \\
\hline 20 & 0 & 0 & 0 & 66.86 & 59.96 \\
\hline
\end{tabular}

Table 3. Estimates of the coefficients of Equation 4 and 5 together with their Standard Errors (SE) and p-values (p)

\begin{tabular}{llrcc}
\hline Coefficient & Term & Value & SE & $\mathrm{p}$ \\
\hline Bracts & & & & $<.995$ \\
$\beta_{0}$ & - & 66.013 & 1.204 & $<0.0001$ \\
$\beta_{1}$ & $\mathrm{~T}$ & 3.107 & 1.204 & $<034$ \\
$\beta_{2}$ & $\mathrm{E}$ & 13.661 & 1.204 & 0.0001 \\
$\beta_{3}$ & $\mathrm{R}$ & 3.054 & 1.687 & $<0.0001$ \\
Stems & & 57.242 & 1.120 & 0.004 \\
$\beta_{0}$ & - & 4.150 & 1.120 & $<0.0001$ \\
$\beta_{1}$ & $\mathrm{~T}$ & 12.680 & 1.120 & 0.146 \\
$\beta_{2}$ & $\mathrm{E}$ & 1.763 & 1.090 & $<0.0001$ \\
$\beta_{3}$ & $\mathrm{R}$ & -7.166 & 1.090 & 0.012 \\
$\beta_{11}$ & $\mathrm{~T} \times \mathrm{T}$ & 3.317 & 1.090 & 0.207 \\
$\beta_{22}$ & $\mathrm{E} \times \mathrm{E}$ & 1.470 & 1.090 & 0.951 \\
$\beta_{33}$ & $\mathrm{R} \times \mathrm{R}$ & -0.091 & 1.463 & 0.838 \\
$\beta_{12}$ & $\mathrm{~T} \times \mathrm{E}$ & -0.306 & 1.463 & 0.044 \\
$\beta_{13}$ & $\mathrm{~T} \times \mathrm{R}$ & -3.376 & & \\
\hline
\end{tabular}

Examination of Table 3 reveals that, for bracts, the model coefficients were all significant at the $95 \%$ confidence level. In contrast, in the case of stems, there were four non-significant coefficients $\left(\beta_{3}, \beta_{33}\right.$, $\beta_{12}$ and $\left.\beta_{13}\right)$. The non-significant coefficients were therefore eliminated and a new reduced model was developed. The term containing $\beta_{3}$, although nonsignificant, was retained in the model to ensure hierarchy, being the coefficient $\beta_{23}$ significant. The new coefficients were re-estimated from the experimental data, leading to the following final Equation 6 and 7, expressed in terms of uncoded factors:

$$
\begin{aligned}
& y=17.049+0.311 T+0.455 E+0.305 R \\
& y=-174.81+7.727 T+0.225 E+0.851 R- \\
& 0.073 T^{2}+0.003 E^{2}-0.011 E \cdot R
\end{aligned}
$$

Representative graphs of the resulting response surfaces are displayed in Fig. 2, where the model response (y) is plotted as a function of two factors varying in the cubic part of the design while setting the third to its center-point value. 


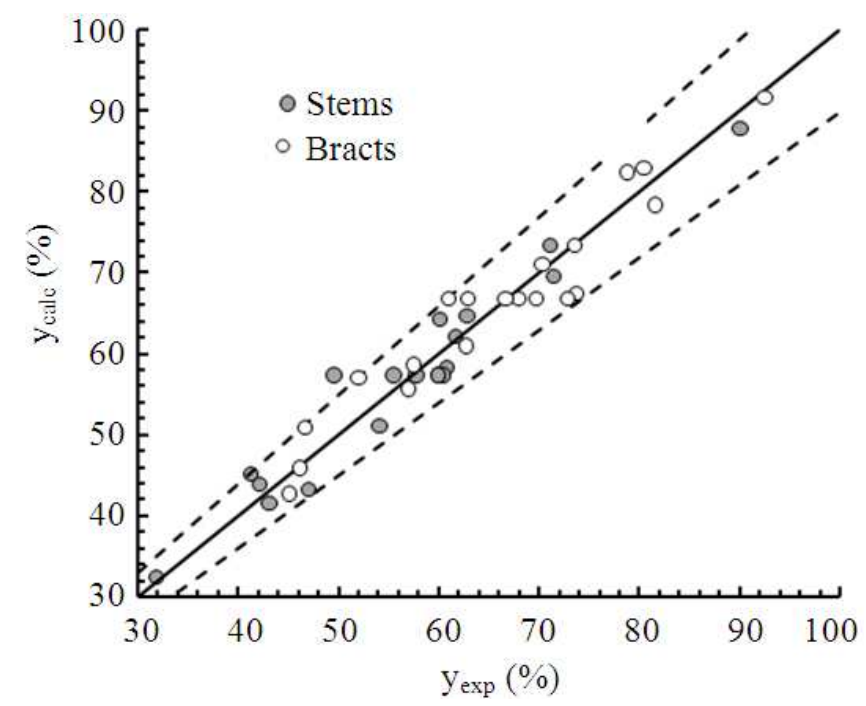

Fig. 1. Comparison between experimental and calculated yields. The dashed lines delimit the $\pm 10 \%$ deviation band

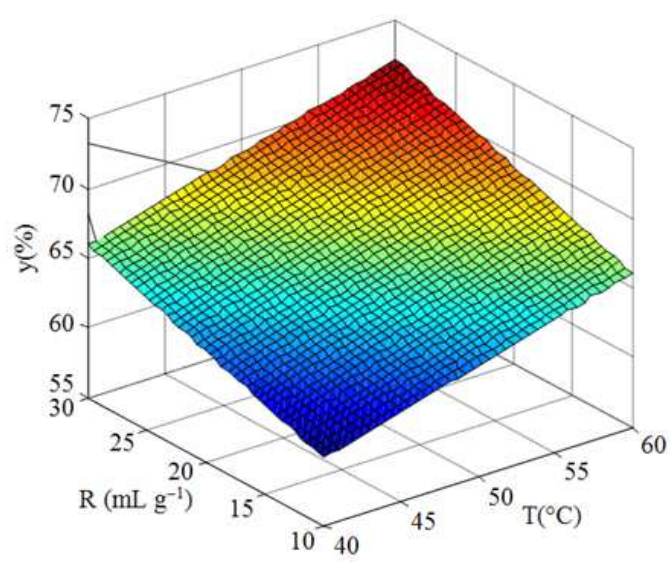

(a)

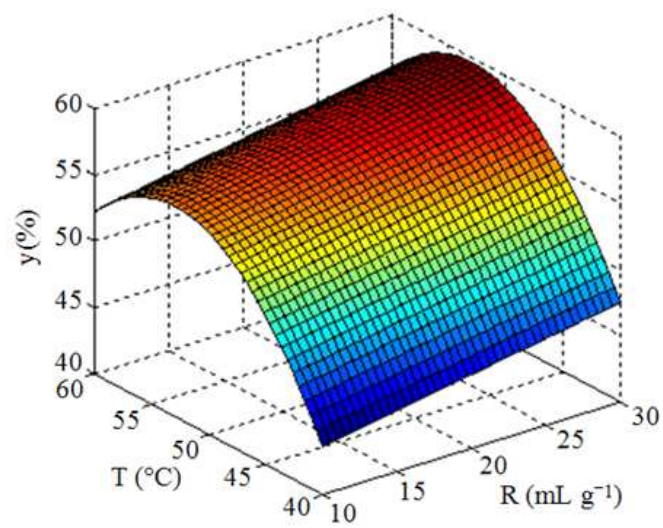

(b)

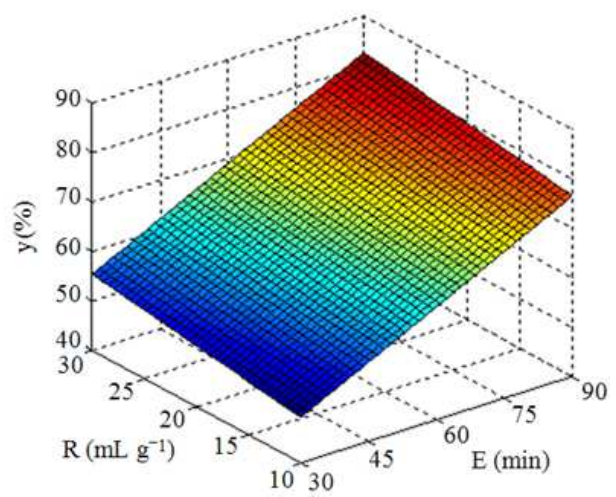

(b)

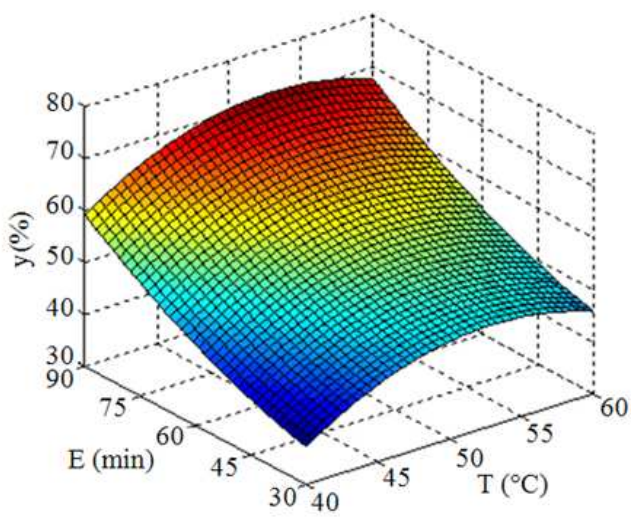

(c)

Fig. 2. Response surface plots showing the influence of T, E and R on polyphenol extraction yields from artichoke bracts (a, b) and stems (c, d) 


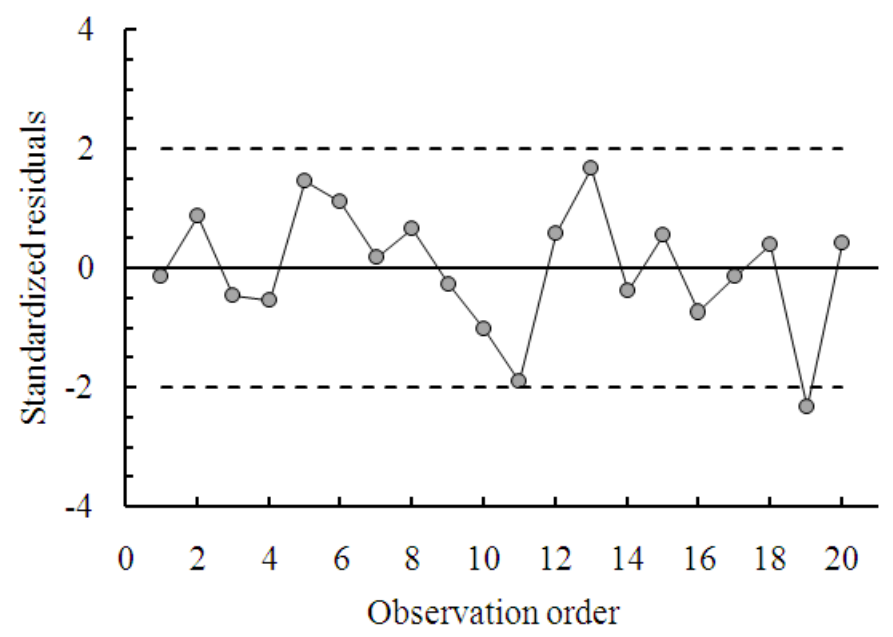

Fig. 3. Standardized residuals Vs. observation order for artichoke stems

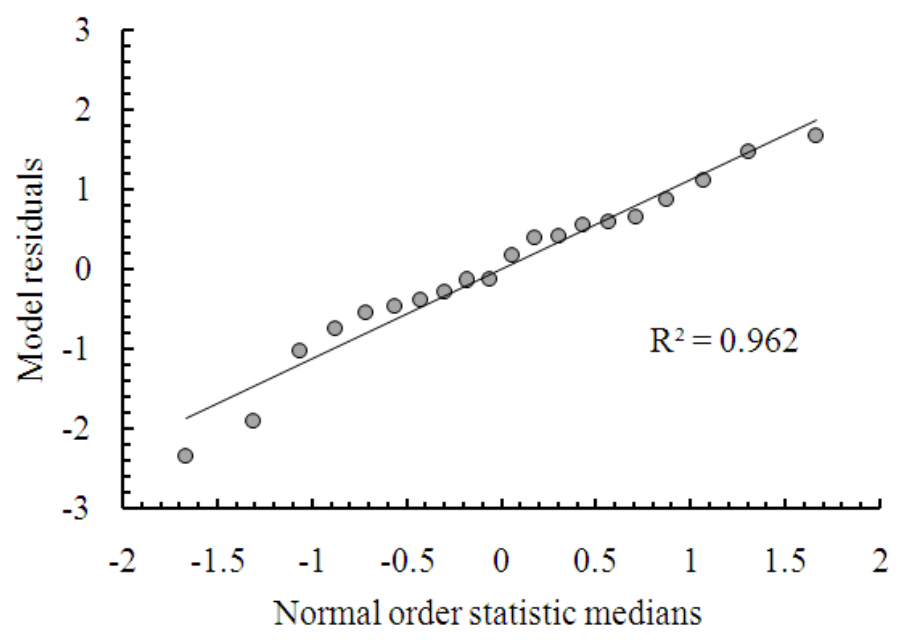

Fig. 4. Normal probability plot showing the trend of ordered model residuals against normal order statistic medians for artichoke stems

Table 4. Results of validation experiments. $\mathrm{y}_{\text {pred }}$ is the predicted model response and $\mathrm{y}_{\mathrm{obs}}$ the experimental value

\begin{tabular}{llccll}
\hline Waste & $\mathrm{T}\left({ }^{\circ} \mathrm{C}\right)$ & $\mathrm{E}(\mathrm{min})$ & $\mathrm{R}\left(\mathrm{mL} \mathrm{g}^{-1}\right)$ & $y_{\text {pred }}$ & $y_{\text {obs }}$ \\
\hline Bracts & 35 & 20 & 6.5 & 39.02 & 40.26 \\
& 63 & 20 & 33.0 & 55.81 & 57.34 \\
\multirow{3}{*}{ Stems } & 63 & 100 & 6.5 & 84.12 & 81.45 \\
& 35 & 20 & 6.5 & 16.01 & 15.22 \\
& 63 & 20 & 33.0 & 48.78 & 50.15 \\
& 63 & 100 & 6.5 & 73.14 & 71.63 \\
\hline
\end{tabular}

The reduced model described by Equation 7 was checked for statistical significance. The standardized residuals, defined as the deviations from the mean expressedin terms of standard errors, were calculated and plotted against the formal order of runs (Fig. 3). The resulting data points were randomly scattered around the zero value and, with the exception of one outlier, within the \pm 2 band. The normal probability plot, obtained by plotting the ordered model residuals against normal order statistic medians, exhibited a linear trend, with a 
coefficient of determination $\mathrm{R}^{2}=0.962$ (Fig. 4). It can, therefore, be concluded that the reduced model is statistically significant and can be used to evaluate the influence of process parameters on the recovery of polyphenols from artichoke bracts.

Finally, to assess the prediction capabilities of the two models, additional experiments were performed in points outside the factorial region of the design. Table 4 shows the observed and predicted extraction yields. As can be seen, experimental and calculated values were in fairly good agreement, the average percent differences being $3.04 \%$ for bracts and $3.27 \%$ for stems.

\section{DISCUSSION}

The first point emerging from the results presented here is that artichoke waste is a good source of phenolic compounds and that stems are richer in polyphenols than bracts. However, it should be emphasized that the polyphenol content of artichoke waste is not only dependent on the plant part considered and the genotype (Pandino et al., 2011), but also on the conditions of the blanching process. Blanching is a thermal treatment used to minimize the deterioration of vegetables products during storage (Nayak et al., 2013). Generally, it is carried out by immersing the plant material in hot water for a time sufficient to inactivate the enzymes responsible for undesired chemical reactions. In the case of artichoke, blanching is primarily performed to inactivate polyphenol oxidase. This enzyme catalyzes the oxidation of orthodihydroxyphenolic substrates, such as caffeoylquinic esters, causing the occurrence of browning phenomena and flavor development (Lattanzio et al., 2009). In addition to avoid degradation of the phenolic compounds that are already present in artichoke tissues, blanching can also lead to the formation of new polyphenols (Lutz et al., 2011). Since part of these compounds are released into the blanching water, the net effect on polyphenols will depend on their initial amount and the severity of the treatment. Thus, if one wants to use artichoke waste as a source of natural antioxidants, the blanching process should be optimized to maximize the amount of polyphenols retained in the waste.

Another interesting result of this study is that all of the process variables considered, that is, Temperature (T), Extraction time (E) and liquid-to-solid Ratio (R), affected the recovery of polyphenols from artichoke bracts and stems, but not in the same way.

In the case of bracts, the yield of polyphenol extraction was affected linearly by the three factors, leading to the planar response surfaces shown in Fig. 2a and $\mathbf{b}$. Since the model described by Equation 6 contains only linear terms, it follows that an increase in any of the independent variables would increase the extraction yield, whatever the values of the remaining ones. On the basis of the values of the associated coefficients (see Table 3), the following influential order can be established: $\mathrm{E}>\mathrm{T}>\mathrm{R}$. However, due to the higher value of $\beta_{2}$, compared to $\beta_{1}$ and $\beta_{3}$, increasing the extraction time will be more effective than increasing the temperature or the liquid-to-solid ratio.

Experiments on stems revealeda more complex dependence of the process response on the three main factors. In particular, the linear coefficients decreasedin the same order as observed for bracts $(E>T>R)$, but second-order and interaction terms were also present. The quadratic dependence on temperature was responsible for the curvature of the resulting response surfaces (Fig. 2c and d). Up to about $55^{\circ} \mathrm{C}$, there was a positive effect of temperature, which can be attributed to the positive influence of this factor on the kinetics of polyphenol extraction from the plant material. At higher temperatures, however, the extraction yield decreased, which is probably due to heat-induced degradation processes (Volf et al., 2014), thereby resulting in an apparent reduction of extraction yields.

As regards the extraction time, in addition to the already described linear effect, there was a positivequadratic effect and a negative interaction with the liquid-to-solid ratio (Fig. 2c and d). This means that, at a given temperature, the influence of the extraction time on the yield is more pronounced at lower liquid-to-solid ratios. The reason may be that atlow liquid-to-solid ratios the mass-transfer of tissue-bound polyphenols into the solvent is reduced (Cussler, 2009), so that the positive effect of extraction time becomes more prominent.

Overall, the results obtained indicate that the extraction time is the most influential factor and that it positively affects the recovery of polyphenols. Also the temperature and the liquid-to-solid ratio have a positive effect on the extraction yield, but the former should be set properly to avoid thermal degradation of phenolic compounds.

The observed differences in the extraction behavior of bracts and stems are very likely a reflection of the different structural features of the two plant tissues and/or their different phenolic composition (Pandino et al., 2011; Negro et al., 2012). When considering the waste produced from the industrial processing of artichokes, containing bracts and stems in different proportions, results closer to those found for the one or the other component can be expected, depending on the relative amount of the two components. 


\section{CONCLUSION}

The results of this study indicate that the two main components of artichoke waste, the outer bracts and the stems, are a rich source of polyphenols. It has also been shown that these compounds can be easily recovered from the plant material by an environmentally friendly solvent-extraction procedure and that the response surface methodology provides an efficient way to evaluate the influence of process variables on the extraction yields.

Future research should be directed at investigating the extraction behavior of the real industrial waste so as to highlight possible differences with the results found for the two waste components. The feasibility of redesigning the blanching process to minimize the loss of polyphenols in the blanching water could be another interesting issue to be addressed in future studies.

\section{ACKNOWLEDGEMENT}

The researcher gratefully thanks Dr. Gianluca Maffei for his assistance in the experimental work.

\section{REFERENCES}

Cussler, E.L., 2009. Diffusion: Mass Transfer in Fuid Systems. 3rd Edn, Cambridge University Press, Cambridge, ISBN-10: 0521871212, pp: 631.

Fabbri, A., S. Serranti and G. Bonifazi, 2014. Biochemical Methane Potential (BMP) of artichoke waste: the inoculum effect. Waste Manage. Res., 32: 207-214. DOI: $10.1177 / 0734242 X 14521680$

FAOSTAT, 2011. Food and agricultural commodities production, Final 2011 Data,

Gaafar, A.A. and Z.A. Salama, 2013. Phenolic compounds from artichoke (Cynara scolymus L.) by products and their antimicrobial activities. J. Biol. Agric. Healthcare.

Kosseva, M.R., 2009. Processing of food wastes. Adv. Food Nutr. Res., 58: 57-136. DOI: 10.1016/S10434526(09)58003-5

Lattanzio, V., P.A. Kroon, V. Lonsalata and A. Cardinali, 2009. Globe artichoke: A functional food and source of nutraceutical ingredients. J. Funct. Foods, 1: 131-134. DOI: 10.1016/j.jff.2009.01.002

López-Molina, D., M.D. Navarro-Martínez, F. Rojas Melgarejo, A.N. Hiner and S. Chazarra et al., 2005. Molecular properties and prebiotic effect of inulin obtained from artichoke (Cynara scolymus L.). Phytochemistry, 66: 1476-1484. DOI: 10.1016/j.phytochem.2005.04.003
Lutz, M., C. Henriquez and R. Escobar, 2011. Chemical composition and antioxidant properties of mature and baby artichokes (Cynara scolymus L.), raw and cooked. J. Food Compos. Anal., 24: 49-54. DOI: 10.1016/j.jfca.2010.06.001

Montgomery, D.C., 2008. Design and Analysis of Experiments, 7th Edn., John Wiley and Sons, New York, ISBN-10: 0470128666. pp: 680.

Nayak, B., R.H. Liu and J. Tang, 2013. Effect of processing on phenolic antioxidants of fruits, vegetables and grains-A review. Crit. Rev. Food Sci. Nutr. DOI: 10.1080/10408398.2011.654142

Negro, D., V. Montesano, S. Grieco, P. Crupi and G. Sarli et al., 2012. Polyphenol compounds in artichoke plant tissues and varieties. J. Food Sci., 77: C244-C252. DOI: $10.1111 / \mathrm{j} .1750-$ 3841.2011.02531.x

Pandino, G., S. Lombardo and G. Mauromicale, 2013. Globe artichoke leaves and floral stems as a source of bioactive compounds. Ind. Crop. Prod., 44: 44-49. DOI: 10.1016/j.indcrop.2012.10.022

Pandino, G., S. Lombardo, G. Mauromicale and G. Williamson, 2011. Profile of polyphenols and phenolic acids in bracts and receptacles of globe artichoke (Cynara cardunculus var. scolymus) germplasm. J. Food Compos. Anal., 24: 148-153. DOI: $10.1016 /$ j.jfca.2010.04.010

Panusa, A., A. Zuorro, R. Lavecchia, G. Marrosu and R. Petrucci, 2013. Recovery of natural antioxidants from spent coffee grounds. J. Agric. Food Chem., 61: 4162-4168. DOI: 10.1021/jf4005719

Raccuia, S.A., M.G. Melilli, P. Calderaro and S. Scandurra, 2013. Globe artichoke genetic variability for residual biomass production as renewable resources of energy in South Italy. Acta Hortic., 983: 129-132.

Sergio, L., A. De Paola, V. Linsalata, A. Cardinalia and S. Vanadia et al., 2010. The use of artichoke peroxidase to remove phenols from olive mill waste water. Fresenius Environ. Bull., 19: 3028-3036.

Volf, I., I. Ignat, M. Neamtu and V.I. Popa, 2014. Thermal stability, antioxidant activity and photooxidation of natural polyphenols. Chem. Pap., 68: 121-129. DOI: 10.2478/s11696-013-0417-6

Wang, L.J., 2013. Production of bioenergy and bioproducts from food processing wastes: A review. Trans. ASABE, 56: 217.229.

Zuorro, A. and R. Lavecchia, 2011. Polyphenols and energy recovery from spent coffee grounds. Chem. Eng. Trans., 25: 285-290. DOI: 10.3303/CET1125048 
Zuorro, A. and R. Lavecchia, 2012. Spent coffee grounds as a valuable source of phenolic compounds and bioenergy. J. Clean. Prod., 34: 49-56. DOI: 10.1016/j.jclepro.2011.12.003

Zuorro, A. and R. Lavecchia, 2014. Recovery of phenolic antioxidants from the peel fraction of bilberry (Vaccinium myrtillus L.) processing waste. Biotechnol. AIJ, 9: 273-279.

Zuorro, A., M. Fidaleo and R. Lavecchia, 2013a. Response Surface Methodology (RSM) analysis of photodegradation of sulfonated diazo dye Reactive Green 19 by UV/H2O2 process. J. Environ. Manage., $\quad$ 128: 28-35. DOI: 10.1016/j.jenvman.2013.04.023
Zuorro, A. and R. Lavecchia, 2013. Influence of extraction conditions on the recovery of phenolic antioxidants from spent coffee grounds. Am. J. Applied Sci., 10: 478-486. DOI: 10.3844/ajassp.2013.478.486

Zuorro, A., R. Lavecchia, F. Medici and L. Piga, 2013b. Enzyme-assisted production of tomato seed oil enriched with lycopene from tomato pomace. Food Bioproc. Technol., 6: 3499-3509. DOI: 10.1007/s11947-012-1003-6 